\title{
Course of relational and non-relational recognition memory across the adult lifespan
}

\author{
Eleonore Soei ${ }^{1,2,3}$ and Irene Daum ${ }^{1,2}$ \\ ${ }^{1}$ Institute of Cognitive Neuroscience, Department of Neuropsychology, Ruhr-University Bochum, 44780 Bochum, Germany; \\ ${ }^{2}$ International Graduate School of Neuroscience, Ruhr-University Bochum, 44780 Bochum, Germany
}

\begin{abstract}
Human recognition memory shows a decline during normal ageing, which is thought to be related to age-associated dysfunctions of mediotemporal lobe structures. Whether the hippocampus is critical for human general relational memory or for spatial relational memory only is still disputed. The human perirhinal cortex is thought to be critically involved in non-relational memory, but another view postulates hippocampal involvement in both relational and non-relational memory. Investigating whether there is a differential impact of ageing on these memory processes may shed further light into these issues. Thus, in the present study, 106 healthy adults performed three recognition memory tasks in a consecutive age groups design involving a range from age 20 to 76 . This allowed the separate assessment of spatial and nonspatial relational memory as well as non-relational memory. Both spatial and nonspatial relational memory declined in the $66-76 \mathrm{yr}$ group. This pattern is consistent with the presumed course of hippocampal changes across normal ageing and points to the hippocampal role in relational memory in general. An impairment of non-relational memory commenced earlier in the 51-65 yr group. This finding is discussed in relation to perceptual/attentional mediation of memory and its potential brain correlates in ageing.
\end{abstract}

There is an ongoing debate about the contribution of the hippocampus to relational memory (Eichenbaum 2004) as compared to spatial relational processing consistent with the cognitive map theory (O'Keefe 1999). Studies of hippocampal lesions in humans and fMRI studies generally support the hypothesis of a general role of the hippocampus in relational memory (Spiers et al. 2001; Eldridge et al. 2005; Fenker et al. 2005; Kumaran et al. 2007). Right hippocampus activation correlated with novelty detection of both spatial and nonspatial relations of stimulus pairs, but not with novelty detection of single stimuli, which in turn correlated with perirhinal activation (Kohler et al. 2005). In contrast, animal lesion studies and human fMRI studies focusing mainly on spatial localization and navigation (Maguire et al. 1997, 1998; O'Keefe 1999; Burgess et al. 2002; Kumaran and Maguire 2005; Lavenex et al. 2006) have supported the idea of a more important role of the hippocampus in spatial compared to nonspatial relational processing.

Relational memory has generally been linked to recollection, i.e., episodic memory for event features within a specific context whereas item or non-relational memory is thought to rely on familiarity, i.e., the feeling of knowing an item without remembering the study context (Yonelinas 2002; Rugg and Yonelinas 2003; Aggleton and Brown 2006). Evidence for the dissociability of relational and item/non-relational recognition stems from fMRI studies of differential hippocampus vs. perirhinal cortex/anterior parahippocampal cortex activations associated with the two types of memory (Pihlajamaki et al. 2004; Kohler et al. 2005; Daselaar et al. 2006; Montaldi et al. 2006). Studies of patients with hippocampal lesions have as yet not yielded a clear picture with respect to the dissociability of relational and non-relational memory (Yonelinas et al. 2002; Manns et al. 2003; Mayes et al. 2004; Wixted and Squire 2004; Wais et al. 2006; Wixted 2007). Interestingly, single-unit recordings in human epilepsy patients demonstrated that hippocampal neurons

${ }^{3}$ Corresponding author.

E-mail Eleonore.Soei@rub.de; fax 49-234-14622.

Article is online at http://www.learnmem.org/cgi/doi/10.1101/lm.757508. show a familiarity response in the absence of successful recollection, but notably also in the absence of successful non-relational recognition (Rutishauser et al. 2006). Thus, whether the hippocampus is involved in recollection/relational memory only or in both recollection/relational and familiarity/non-relational memory remains to be clarified. The perirhinal cortex was shown to be involved in the perceptual analysis of single items and in binding of individual stimulus features into a coherent representation of an object, which requires perceptual and mnemonic competence (Bussey et al. 2002; Buckley 2005; Bussey and Saksida 2005). It may support relational processing under some circumstances, such as unitizing two features of the same kind to one single item or merged unity representation, but not spatiotemporal relational associations (Norman and O'Reilly 2003; Mayes et al. 2004; Jager et al. 2006). The hippocampus, on the other hand, mediates associations of the same kind such as object-object associations (Kesner and Hopkins 2006).

To further clarify the issues of hippocampal involvement in spatial relational vs. general relational memory and the potential dissociability of recollection/relational memory and familiarity/ non-relational memory, this study investigates effects of healthy ageing on different recognition memory processes. Hippocampal volume has been found to decline in a curvilinear manner with rising age, with an accelerated decrease after age 50-60 (Raz et al. 2005; Cohen et al. 2006). The volume of the perirhinal cortex remains relatively stable across age (Insausti et al. 1998a,b). Consistent with these findings, recollection/relational memory showed a more pronounced age-related decline than the relatively stable familiarity/non-relational memory component of recognition memory in previous studies (Grady et al. 1994; Yonelinas 2002; Cabeza et al. 2004). However, this pattern has been challenged in a recent study (Prull et al. 2006). Using three different methods to estimate recollection and familiarity, recollection was consistently found to be reduced in older compared to younger subjects, irrespective of the analysis method. Two of the three methods did, however, also yield familiarity deficits in older adults. Support for an age-related decline of both recognition memory components also came from another recent study 
involving 144 young and old participants (Toth and Parks 2006). The reasons for these conflicting findings are as yet unresolved. The ageing studies carried out so far were exclusively based on extreme group comparisons of one young and one older group, a procedure which entails the known problems of cohort effects. A consecutive age groups design might be better suited to elucidate the course of progression of relational and non-relational memory across ageing.

To address the aforementioned issues, spatial and nonspatial relational as well as non-relational memory were studied in a consecutive age groups design, involving healthy subjects ranging in age from 20 to $76 \mathrm{yr}$. A variant of a task which yielded specific hippocampal and perirhinal cortex activations in a recent fMRI study was used (Kohler et al. 2005). Despite the known limitations of crosssectional investigations (Hedden and Gabrieli 2004), the design allows an estimation of the course of the progression of recognition memory across age and may mirror the course of the presumed age-associated changes in different brain regions. For instance, the presumed curvilinear changes in hippocampal function may be paralleled by a late-life decline in relational memory. The separate assessment of spatial and nonspatial relational memory should add to our knowledge about the generality of the hippocampal mediation of relational memory. If the perirhinal cortex was critical for non-relational memory, its estimates should remain relatively stable with rising age. A critical involvement of the hippocampus in non-relational memory, on the other hand, would yield a similar decline of relational and nonrelational memory.

\section{Results}

The data for neuropsychological screening are summarized in Table 1 . The age groups differed significantly in estimated intellectual ability (IQ) $\left(F_{(3,102)}=3.48, P=0.019\right)$, with better performance of the $66-76$ yr group $(P=0.046)$ and the $51-65$ yr group $(P=0.037)$ compared to the $20-35$ yr group. The groups did not differ significantly on verbal short-term memory (both $F \leq 2.29$ ). There were, however, significant age differences for nonverbal memory. The group difference in nonverbal short-term memory $\left(F_{(3,102)}=11.47, P<0.001\right)$ was due to the poorer performance of the 66-76 yr group compared to the $20-35$ yr group $(P<0.001)$ and the $36-50$ yr group $(P<0.001)$. Analysis of nonverbal working memory also yielded significant age differences $\left(F_{(3,102)}=12.34, P<0.001\right)$, with lower scores of the 66-76 $\mathrm{yr}$ group compared to the $20-35 \mathrm{yr}(P<0.001)$ and the $36-50 \mathrm{yr}$ groups $(P<0.001)$, and lower scores of the 51-65 yr group compared to the $20-35$ yr group $(P=0.006)$. The four age groups differed neither on depression $(F=1.70)$ nor the dementia screening score (MMST [mini-mental status test], $F=2.09$ ). Taken together, the data suggest typical patterns observed in healthy ageing samples.

\section{Spatial relational memory in the four age groups comparison \\ ANOVA yielded significant age differences in discrimination in- dices $\left(F_{(3,102)}=11.01, P<0.001\right.$, see Fig. 1$)$. Post-hoc Bonferroni- corrected tests yielded the following results, and no other group differences reached significance. The 66-76 yr group showed poorer discrimination than each of the younger groups (20-35 yr}

group $[P<0.001], 36-50$ yr group $[P=0.001], 51-65$ yr group $[P=0.010])$. Separate analysis of the hit rates yielded the same pattern (see Fig. 2, overall group difference $F_{(3,102)}=10.58$, $P<0.001,66-76$ group vs. $20-35$ yr group $[P<0.001]$, vs. $36-50$ yr group $[P=0.001]$, vs. $51-65$ yr group $[P=0.020])$. The significant age group difference for false alarm rates $\left(F_{(3,102)}=4.19\right.$, $P=0.008$ ) was due to higher error rates in the 66-76 yr group compared to the youngest group $(P=0.004$, see Fig. 2$)$. Analysis of response bias did not yield significant age group differences (see Table $2, F=0.79$ ).

RTs associated with hits (see Fig. 3) yielded significant age differences $\left(F_{(3,102)}=9.58, P<0.001\right)$, with the $20-35$ yr group responding faster than each of the other groups (36-50 yr group $[P=0.036], 51-65$ yr group $[P=0.025], 66-76$ yr group $[P<0.001])$, and the $36-50 \mathrm{yr}$ group responding faster than the 66-76 yr group $(P=0.047)$. The significant RT difference on false alarm trials $\left(F_{(3,102)}=3.26, P=0.025\right)$ was due to faster responses of the youngest compared to the oldest group $(P=0.028)$.

\section{Nonspatial relational memory in the four age groups comparison}

As in the spatial task, analysis of the discrimination indices for nonspatial memory (see Fig. 1 ) indicated a significant age difference $\left(F_{(3,102)}=8.47, P<0.001\right)$, with the $66-76 \mathrm{yr}$ group showing poorer recognition performance than each of the younger groups

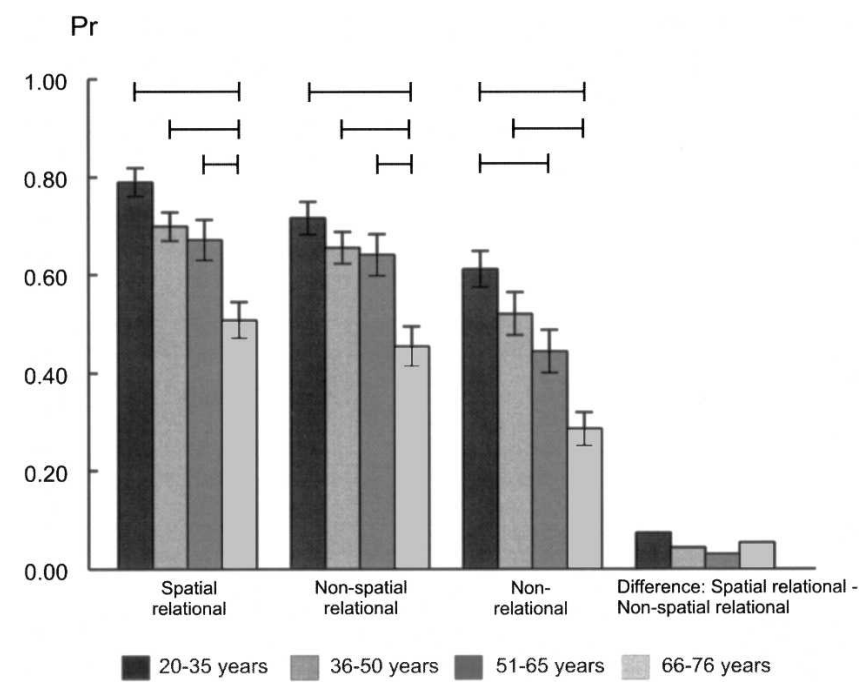

Figure 1. Discrimination indices ( $M$, means; SEs, standard errors) of spatial and nonspatial relational and non-relational memory estimates in the four age groups. Lines indicate significant paired group differences (Bonferroni-corrected). 


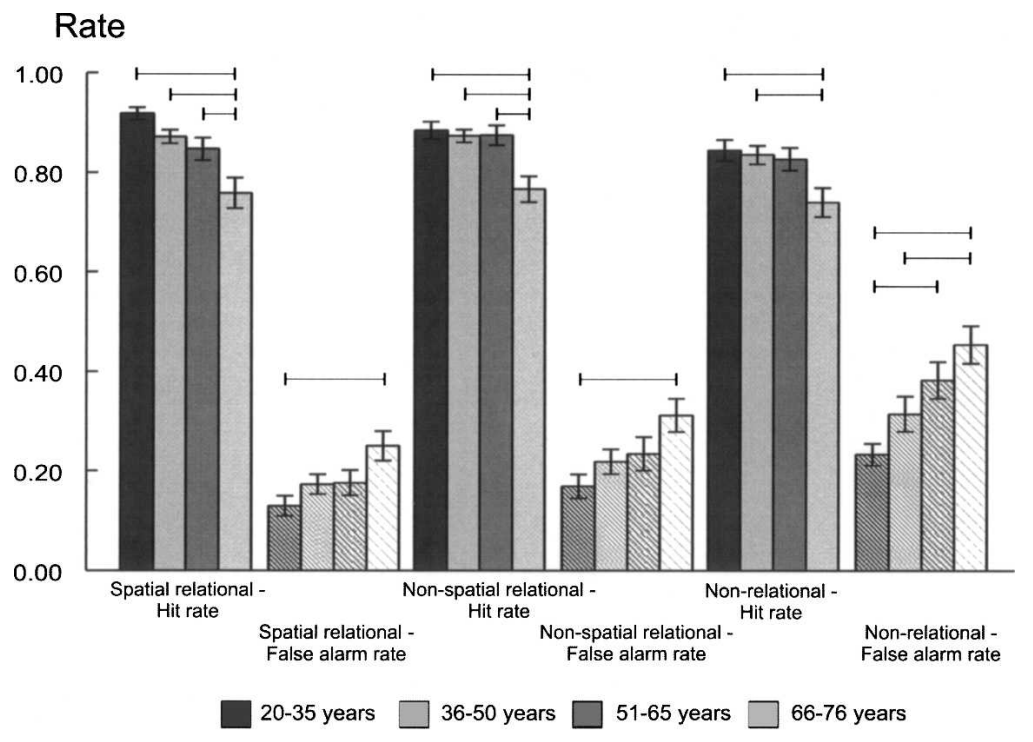

Figure 2. Hits and false alarm rates ( $M$, means; SEs, standard errors) of spatial and nonspatial relational and non-relational memory estimates in the four age groups. Lines indicate significant paired post-hoc tests for group differences (Bonferroni-corrected).

(20-35 yr group $[P<0.001], 36-50$ yr group $[P=0.002)$, and the $51-65$ yr group $[P=0.006])$. Analysis of hit rates yielded the same pattern (overall group difference $F_{(3,102)}=7.85, P<0.001,66-76$ group vs. $20-35$ yr group $[P<0.001]$, vs. $36-50$ yr group $[P=0.001]$, vs. $51-65$ yr group $[P=0.001])$. Similar to the spatial task, the false alarm group difference $\left(F_{(3,102)}=3.98, P=0.010\right)$ was due to higher false alarm rates in the $66-76$ yr group compared to the youngest group $(P=0.005)$ (see Fig. 2$)$. Response bias estimates did not differ between age groups (see Table 2, $F=0.51)$.

The significant RT difference on hits trials (see Fig. 3, $\left.F_{(3,102)}=8.02, P<0.001\right)$ was due to slower responses in the 66$76 \mathrm{yr}$ relative to the two youngest age groups (20-35 yr group $[P<0.001], 36-50$ yr group $[P=0.021])$. The group difference for RTs on false alarms trials $\left(F_{(3,102)}=7.01, P<0.001\right)$ was due to faster RTs of 20-35 yr group compared to the 66-76 yr group $(P<0.001)$ and the $36-50$ yr group $(P=0.019)$.

\section{Non-relational memory in the four age groups comparison}

The significant age group difference for the discrimination index $\left(F_{(3,102)}=11.01, P<0.001\right)$ was due to a poorer performance of the 66-76 yr group relative to the two youngest age groups (vs. $20-35$ yr group $[P<0.001]$, vs. $35-50$ yr group $[P=0.001])$. The difference between the 66-76 yr and 51-65 yr groups approached but did not reach significance $(P=0.055)$. In addition, the discrimination index of the 51-65 yr group was lower than the index of the youngest group $(P=0.023)$ (see Fig. 1$)$. Analysis of hit rates also yielded an overall group difference $\left(F_{(3,102)}=4.15, P=0.008\right)$, with lower hit rates of the 66-76 yr group compared to the two youngest age groups (vs. 20-35 yr group $[P=0.012]$, vs. $36-50$ yr group $[P=0.025]$, see Fig. 2). The significant age difference in false alarm rates $\left(F_{(3,102)}=7.81, P<0.001\right)$ was due to higher rates of the $66-76 \mathrm{yr}$ group relative to the $20-35$ yr group $(P<0.001)$ and the $36-50$ yr group $(P=0.026)$. In addition, the false alarm
Table 2. Response biases (M, means; SEs, standard errors) for the four age groups

\begin{tabular}{|c|c|c|c|c|c|c|c|c|}
\hline & \multicolumn{2}{|c|}{$20-35 \mathrm{yr}$} & \multicolumn{2}{|c|}{$36-50 \mathrm{yr}$} & \multicolumn{2}{|c|}{$51-65 \mathrm{yr}$} & \multicolumn{2}{|c|}{$66-85 \mathrm{yr}$} \\
\hline & $M$ & (SEs) & $M$ & (SEs) & $M$ & (SEs) & $M$ & (SEs) \\
\hline $\begin{array}{l}\text { Response bias index spatial } \\
\text { relational memory }\end{array}$ & 0.60 & $(0.04)$ & 0.56 & $(0.03)$ & 0.54 & $(0.04)$ & 0.51 & $(0.05)$ \\
\hline $\begin{array}{l}\text { Response bias index nonspatial } \\
\text { relational memory }\end{array}$ & 0.59 & $(0.04)$ & 0.61 & $(0.03)$ & 0.63 & $(0.04)$ & 0.56 & $(0.04)$ \\
\hline $\begin{array}{l}\text { Response bias index } \\
\text { non-relational memory }\end{array}$ & 0.62 & $(0.03)$ & 0.65 & $(0.03)$ & 0.69 & $(0.04)$ & 0.63 & $(0.04)$ \\
\hline
\end{tabular}

rates of the 51-65 yr group were higher than those of the 20-35 yr group $(P=0.011)$. Response bias estimates did not differ between groups (see Table 2, $F=0.79$ ).

The significant RT difference for hits trials (see Fig. 3, $F_{(3,102)}=5.19$, $P=0.002)$ was related to slower responding of the 66-76 yr group compared to the two youngest groups (vs. 20-35 yr $[P=0.001]$, vs. $36-50$ yr $[P=0.030])$. The RT on false alarm trials did not differ between groups $(F=2.45)$.

\section{Direct comparison of spatial} and nonspatial relational and non-relational memory in the four age groups

An ANOVA with repeated measures including the discrimination ability in the three memory tasks as the withinsubjects factor and age group as the between-factor yielded a main effect of task $\left(F_{(2,102)}=55.84, P<0.001\right)$, as performance in the non-relational memory task is generally lower than in both relational memory tasks. There was also a main effect of age group $\left(F_{(3,102)}=15.86, P<0.001\right)$, showing decreasing discrimination ability with increasing age. However, there was no memory task $\times$ age group interaction $\left(F_{(3,102)}=0.51\right)$.

\section{Relationship between age, neuropsychological data, and discrimination indices of spatial and nonspatial relational and non-relational memory across the adult lifespan}

Correlation analyses, corrected for multiple testing, were conducted with all 106 participants to elucidate the relationship between the neuropsychological data and recognition memory indices across the adult lifespan, and not only between age groups. They yielded negative correlations between age and spatial relational $(R=-0.47, P<0.001)$, nonspatial relational $(R=-0.40$, $P<0.001)$, and non-relational memory $(R=-0.47, P<0.001)$, respectively. Spatial and nonspatial relational memory correlated positively $(R=0.60, P<0.001)$, as did spatial relational and nonrelational memory $(R=0.61, P<0.001)$ and to a relatively lesser extent nonspatial relational and non-relational memory $(R=0.50, P<0.001)$. The same analysis conducted separately for each age group, corrected for multiple testing, did not yield any statistically significant correlation in any of the groups.

All stepwise regression analyses, also conducted across the adult lifespan, yielded significant results, with age alone being a predictor for spatial relational $\left(F_{(1,104)}=28.86, P<0.001\right.$, 


\section{RT}

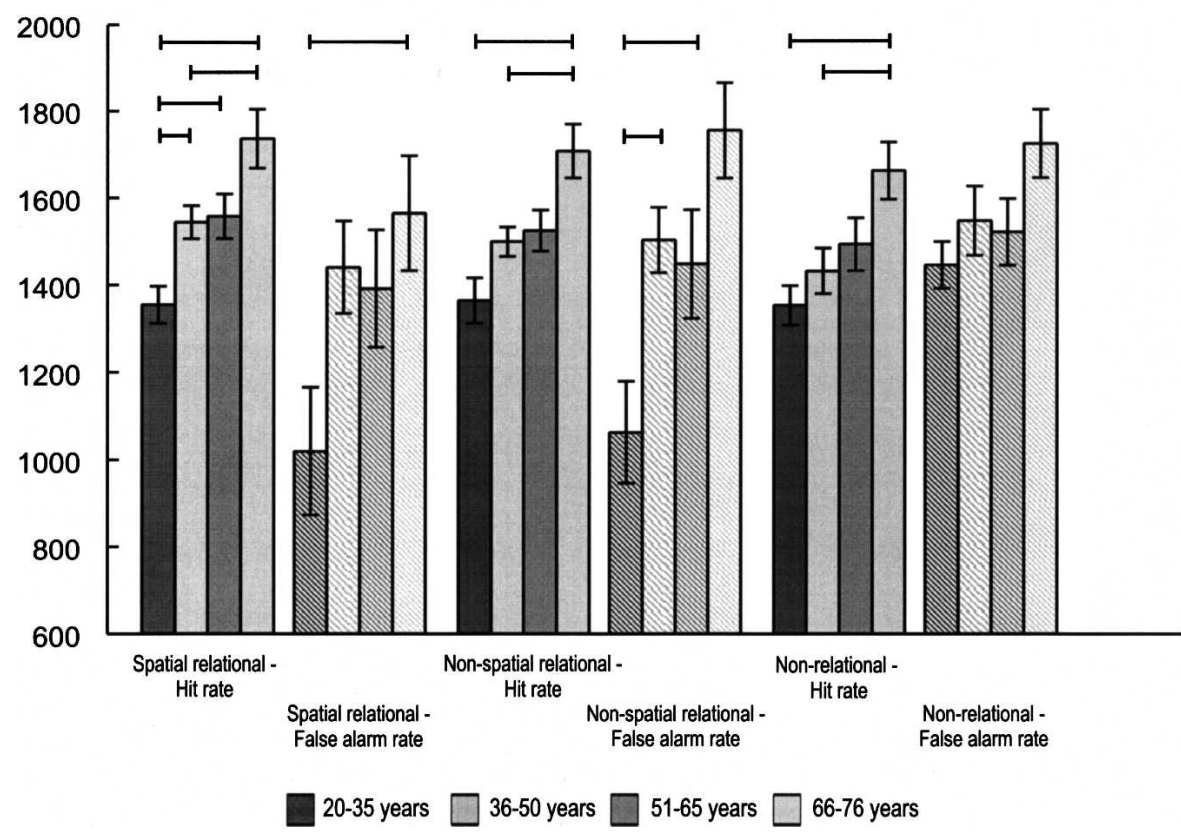

Figure 3. RTs (M, means; SEs, standard errors) for spatial and nonspatial relational and non-relational memory in the four age groups. Lines indicate significant paired post-hoc tests for group differences (Bonferroni-corrected).

$R^{2}=0.22$, Beta-coefficient $\left.=-0.47, T=-5.37, P<0.001\right)$ and non-relational memory $\left(F_{(1,104)}=28.93, P<0.001, R^{2}=0.22\right.$, Beta-coefficient $=-0.47, T=-5.38, P<0.001)$. For nonspatial relational memory, age combined with verbal short-term memory and IQ were included as predictors $\left(F_{(3,102)}=11.29, P<0.001\right.$, $R^{2}=0.25$, Beta-coefficient[age $]=-0.41$, T[age $]=-4.46, P<0.001$, Beta-coefficient[verbal short-term memory] $=0.20$, T[verbal shortterm memory] $=2.29, P=0.024$, Beta-coefficient $[I Q]=0.20$, $T[I Q]=2.17, P=0.032$ ).

\section{Discussion}

The aim of the present study was to assess the course of spatial and nonspatial relational and non-relational memory across the adult age range in healthy subjects ranging in age from 20 to 76 yr. In summary, the declines of discrimination ability in both spatial and nonspatial relational memory had a strikingly similar late onset, as the 66-76 yr group was outperformed by all younger age groups (see Fig. 1). Additionally, spatial and nonspatial relational memory correlated highly across the adult lifespan. Taken together, the analyses of the four age groups and correlational analyses across the lifespan add evidence to the idea that the hippocampus mediates general relational processing. Non-relational memory showed an earlier commencing decline, with subjects in the 51-65 yr age range also showing a discrimination ability deficit relative to the youngest group, and there was also no difference between the two oldest groups (see Fig. 1). This appears consistent with the account of a hippocampal role in both relational and non-relational memory. A closer inspection of hit and false alarm rates revealed that the earlier commencing decline of non-relational memory was mainly due to the high false alarm rates of the two oldest groups relative to the youngest group (see Fig. 2). The patterns of the hit rates across ageing were strikingly similar for spatial and nonspatial relational, but somewhat different for non-relational memory, which speaks in favor of the dissociability of relational and non- relational memory. However, age correlated with and predicted (solely and jointly) performance in all three tasks across the adult lifespan. With respect to the lack of a statistically significant task $\times$ age group interaction, one has to bear in mind that (1) the relational and non-relational tasks were designed to aim at differential memory processes and (2) the non-relational memory task was confounded by a higher level of difficulty, and thus the interpretation of lacking interaction between noncomparable memory performances is not straightforward. Taken together, the implications concerning the issue of dissociability of relational and non-relational memory vs. the issue of hippocampal involvement in non-relational memory may be potentially complicated, and this will be discussed in the second part of the Discussion.

\section{Spatial and nonspatial relational memory indices were affected similarly across ageing}

The relatively late onset of the discrimination impairment for spatial and nonspatial relational memory was based on both lower hit rates and higher false alarm rates in the 66-76 yr group, with relatively stable performance in all younger groups. The question arises as to whether the course of memory changes with rising age would fit with what is known about their neuronal mechanisms and changes with normal ageing. The observed performance pattern appears to mirror neuroimaging data of a late-onset decline of hippocampal volume with a latency (Raz et al. 2005; Cohen et al. 2006). This points to the possibility that the loss of functional integrity required in this task occurred only after a substantial loss of hippocampal volume. However, it should be noted that correlations between hippocampal volume and recollection indices have not been consistently observed (Schiltz et al. 2006). The reduced ability to remember objectlocation associations in older adults has been convincingly linked to reduced anterior hippocampal function (Mitchell et al. 2000). The data do not support the hypothesis that hippocampally mediated relational processing disproportionately affects spatial relationships (O'Keefe 1999). The non-relational memory task used in the present study addressed visuospatial processing to some degree, although clearly less so than the spatial relational memory task, as the participants were instructed to memorize the views of the objects and to later detect changes in the view. These links were illustrated by the findings of the regression analysis across the adult lifespan, with age alone being a strong predictor of the discrimination indices in spatial relational and nonrelational memory and the high correlation between both types of recognition memory. The courses of both spatial and nonspatial relational memory across ageing were, however, strikingly similar, both reflecting a late-life onset of a significant impairment. Taken together, the finding of a strikingly similar course of spatial and nonspatial relational memory across the human adult lifespan supports the idea of a general role of the hippocampus in relational memory processes (Spiers et al. 2001; Eichenbaum 2001, 2004; Kohler et al. 2005; Kesner and Hopkins 2006; Rolls and Kesner 2006; Kumaran et al. 2007) and adds to increasing evidence from experimental animal studies (Gilbert et al. 
2001; Kesner et al. 2002) and human ageing studies using extreme group comparisons (Prull et al. 2006; Toth and Parks 2006).

\section{The non-relational memory index declined earlier across ageing}

A somewhat surprising finding is the relatively early commencing decline of recognition memory and more specifically of the ability to reject nontargets in non-relational memory. This problem was already present in the 51-65 yr group as compared to the youngest group (see Fig. 1), and there were no statistically significant differences in the abilities to discriminate, detect targets, and reject nontargets between the two oldest groups as in relational memory. The present age-related reductions of non-relational memory extend recent evidence of age-associated familiarity deficits in older participants (Prull et al. 2006; Toth and Parks 2006). The two familiarity memory studies were based on extreme groups comparisons (young group age range $=18-28$ vs. older group range $=63-81$ ) (Toth and Parks 2006); (and young group range $=18-22$ vs. older group range $=60-82$ ) (Prull et al. 2006), and thus do not provide information about the course of the age-related changes. The decline of discrimination indices on the non-relational memory task (see Fig. 1) and the lack of memory task $\times$ age group interaction at hand are inconsistent with a previously reported age-associated dissociation of impaired recollection/stable familiarity (Grady et al. 1994; Yonelinas 2002; Cabeza et al. 2004) and accord more with the view that the hippocampus plays a role in both relational and non-relational memory processing (Wixted and Squire 2004; Wais et al. 2006). However, it should be stressed that our non-relational task was clearly more difficult than non-relational recognition based on familiarity of single items, as illustrated on the basis of hit and false alarm rates (see Fig. 2) and RTs (see Fig. 3) (see below for thorough discussion), which limits the comparability of the rates of decline. An important question in this regard is whether non-relational memory of an object consisting of two or more features of the same kind may also depend upon relational memory to some degree (Yonelinas 2002). This is unlikely for the present task, since Norman and O'Reilly (2003) argued that associating two features to one item, but not spatiotemporal relational associations, can be supported by a familiarity-type process, and selective hippocampal lesions spare the recognition for items and also associations between items of the same kind (e.g., word-word or face-face pairs) (Mayes et al. 2004). Still, the current non-relational memory task clearly supported unitization to a lesser degree than, e.g., encoding of arbitrary word pairs in relational vs. non-relational conditions (Quamme et al. 2007). In this study, the strategy to encode arbitrary word pairs as compounds appeared to be clearly related to familiarity memory. The alternative strategy to encode the words as segregated words in a sentence relied on recollection. In our task, the subjects were not instructed to use specific encoding strategies. In the non-relational task, they were simply told to memorize the views, and this may not have sufficed to promote unitization. In summary, this task seemed to place disproportionately high demands on perceptual processing and also did not easily benefit from verbal mediation, semantic processing, or screen position cues as was the case for the relational memory tasks. The non-relational task required the precise perceptual analysis of the conjunctions of features of the two objects as one merged stimulus representation to distinguish the view during recognition. This process has been linked to the perirhinal cortex (Buckley 2005; Bussey and Saksida 2005).

It follows that linking the course of memory changes with rising age in non-relational memory to potential underlying brain mechanisms is more difficult than in the relational memory task. If the present results had been found with equated levels of difficulty between the relational and non-relational tasks, three possible competing scenarios would arise. Firstly, the results would have to be interpreted in favor of the account that the hippocampus is also critical for non-relational memory. Specifically, the decline of the discrimination indices in nonrelational memory and the lacking memory task $\times$ age group interaction could be explained by an ageing-related disruption of hippocampal function. Secondly, the results might be due to an age-associated decline in the functional integrity of the perirhinal cortical circuits despite their stable volume across ageing, which remains to be investigated (Burke and Barnes 2006; Insausti et al. 1998a,b). Unfortunately, the major caveat of the present study is the between-task difference in difficulty, which limits the implications of the results with respect to the first two competing accounts.

A third scenario raises the possibility that the reduction of non-relational memory might be linked to the high attentional demands of the task which might induce the joint recruitment of the perirhinal cortex and the thalamus. There are several lines of evidence which indicate that the non-relational memory task appeared to be more difficult than a simple non-relational memory task based on the familiarity of individual items. It also appeared to be more difficult than the relational tasks, an effect which was not observed in pilot studies. The lower discrimination ability indices for the non-relational memory task were mainly attributable to the higher false alarm rates which increased with each age group (see Fig. 2). The RTs for false alarms were considerably longer for the non-relational compared to the relational memory task; this difference is particularly pronounced for the youngest group, confirming the high level of difficulty (see Fig. 3). It, therefore, may have recruited additional attention-related brain mechanisms which do not play a role in a non-relational memory task using single items, and the brain imaging correlates of the single-item non-relational memory task by Kohler et al. (2005) may not have direct implications for the current study. In the Kohler et al. (2005) study, novel items in the non-relational task were not encountered in the study phase, as opposed to the novel items in the relational tasks, which consisted of objects studied in recombined arrangements. In the current version, we aimed to create similar levels of work load and novelty levels in the relational and non-relational tasks. Taken together, our task also placed higher demands on discriminability, as all non-relational pairs were equally familiar with respect to object co-occurrence and spatial layout, and familiarity of spatial orientation was the key feature for old/novel judgement; hence, the subjects were more inclined to endorse even changed pairs. Given the necessity of recruiting increased perceptual and attentional resources during the non-relational memory task, the question arises whether a recruitment of the thalamus might have been needed in addition to the perirhinal cortex. Familiarity-type recognition has been associated with a circuit linking the perirhinal cortex and the thalamus (Aggleton and Brown 1999). Interestingly, the volume of the thalamus was found to decrease linearly with rising age (Walhovd et al. 2005). Relative to younger subjects, older adults showed increased thalamic activation during verbal encoding or visual target detection, presumably reflecting enhanced attentional processing (Madden et al. 1999, 2004). Higher activation in the thalamus might serve to compensate the reduced occipital activations of older adults shown in response to novel stimuli (Madden et al. 2004) and higher-order visual feature processing in general (Huettel et al. 2001). The age-related posterior-thalamic shift of brain activations may enhance target-relevant features relayed for visual pro- 
cessing of the spatial orientation of the non-relational items (Madden et al. 2004). In contrast, relational memory could be supported by memorizing the relationships of the gist of the objects, without reference to the actual view (i.e., perceptual details) of the objects. Thus, it is possible that the necessity of recruiting increased perceptual and attentional resources during the non-relational memory task might be associated with recruitment of the thalamus. The relatively early memory deficit (slowly beginning in the 51-65 yr group) might be linked to a dysfunction of this mechanism (Raz et al. 2005; Walhovd et al. 2005).

Potential alternative interpretations of the age-associated recognition memory deficits might relate to differences in general level of intellectual status, response speed, or response bias/ motivation. Careful matching and estimating IQ, years of education, MMST, BDI (Beck depression inventory), overall neutral recognition memory biases, and a lenient reaction time window make these explanations unlikely.

In summary, relational and non-relational memory were both significantly affected by age, with a late-life onset of relational memory deficits and an earlier onset of non-relational memory impairment. The pattern across ageing is consistent with a hippocampal mediation of both spatial and nonspatial memory and its late-life dysfunction, and thus strongly supports the account of a hippocampal role in general relational memory (Eichenbaum 2001). A caveat of this study was the unequal task difficulty regarding the relational vs. non-relational memory, thus the current evidence as for the non-relational memory can be interpreted in terms of favoring either one of the following views. Non-relational memory based on perceptually difficult and attention-demanding processes may lead to recognition memory deficits commencing earlier in life, which might be linked to either the critical involvement of the hippocampus in relational and non-relational memory (Wixted and Squire 2004; Wais et al. 2006), loss of functional changes of the perirhinal cortex despite structural changes, or insufficient recruitment of the thalamus.

\section{Materials and Methods}

\section{Participants}

One hundred and six healthy participants were recruited by advertisement and private contact and gave written informed consent to take part in the study. Half of the participants of the youngest age group were undergraduate psychology students and received course credit for participation. The study was conducted in accordance with the requirements of the local ethics committee. Exclusion criteria were a history of neurological, psychiatric, head trauma or substance abuse, a MMST score below 27 , a BDI score above 15, misunderstanding of task instructions as assessed in a post-experimental debriefing, or abnormally long reaction times $(>2500$ $\mathrm{ms})$.

The remaining participants were assigned to one of four age groups: 20-35 yr $(N=28,18$ females, $M=23.89$, $S D=0.78), 36-50$ yr $(N=29,16$ females, $M=42.72, S D=0.75), 51-65 \mathrm{yr}(N=26$, 17 females, $M=55.85, S D=0.81$ ), and 66-76 yr $(N=23,12$ females, $M=69.61$, $S D=0.89$ ).

The proportion of males and females did not differ significantly be- tween groups (all $X^{2}<2.46$ ). Screening of neuropsychological status entailed estimation of general intellectual ability by a short German version of the Wechsler Adult Intelligence Scales (Dahl 1972). Verbal and nonverbal short-term and working memory were assessed by the German version of the digit and block span tests of the Wechsler Memory Scale-Revised (Wechsler 1987).

\section{Assessment of relational and non-relational memory}

The memory assessment procedure of this study was based on a computerized recognition memory test used in a recent fMRI study (Kohler et al. 2005). In brief, subjects had to encode a series of line drawings of object pairs which were presented in distinct spatial layouts (see Fig. 4a). In the test phase, old and novel item pairs were presented, and subjects had to indicate for each pair whether it was previously encountered or novel. Novel items were systematically manipulated versions of the studied pairs involving new object-location (Fig. 4b) or object-object relationships (Fig. 4c).

Non-relational memory was assessed in a variation of the original task. We aimed to develop merged unity representations of object pairings without the need for relational processing. Kohler et al. (2005) used individual objects for the non-relational memory task, which led to ceiling effects in recognition memory. The procedure of the present study aimed to avoid such ceiling effects. The stimuli consisted of two objects presented centrally on the screen (Fig. 4d). Novel items were horizontally flipped versions of the individual objects of the studied pairs, while all test items were intact pairs with regard to their studied spatial layouts (Fig. 4e). Thus, both the object-location and the objectobject relationships were held constant. Critically, as opposed to the two relational tasks, the prior occurrence or familiarity of the spatial orientation determined non-relational recognition memory. In the two relational tasks the spatial orientation of the individual objects never changed. Familiarity of the spatial orientation of the individual objects could thus not contribute to performance. Instead, the prior spatial layout or co-occurrence of the objects had to be remembered for successful task performance. Using paired stimuli rather than individual objects, the
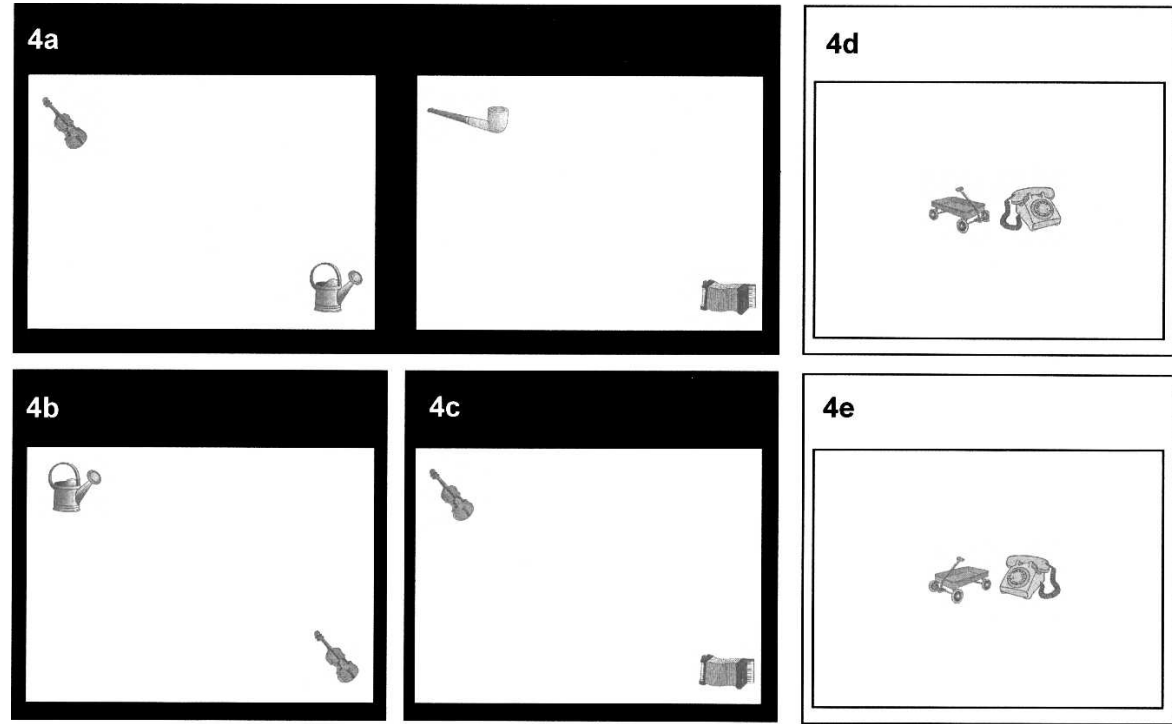

$4 \mathrm{e}$

Figure 4. Schematic overview of the spatial and nonspatial relational (black background) and nonrelational memory (white background) tasks. (a) Example of an original picture from the study phase of the spatial and nonspatial relational task which serves also as an old picture in the test of spatial and nonspatial relational memory. (b) Example of a new picture in the test of spatial relational memory. (c) Example of a new picture in the test of nonspatial relational memory. (d) Example of an original picture from the study phase of the non-relational memory task which serves also as an old picture in the test of non-relational memory. (e) Example of a new picture in the test of non-relational memory. 
general processing load was more comparable in the relational and non-relational tasks. The level of novelty of the distractor items was also more comparable to the relational tasks than in the original version, where the distractors of the non-relational memory task-unlike the relational task-had not been encountered before. In both the relational and non-relational tasks, the novel items were thus systematically altered versions of previously encountered stimuli. The non-relational memory procedure was intended to tap into a merged representation of a single stimulus consisting of two object features.

The number of items in each task and the number of repetitions of the study phase $(N=4)$ were determined on the basis of extensive piloting. The stimulus pairs were based on 144 graylevel texture line drawings of objects presented against a white background; the stimuli were derived from a database (standardized according to naming agreement, familiarity, complexity, and imagery judgements) (Rossion and Pourtois 2004). For each participant, 72 drawings were randomly allocated to the relational task, the remainder to the non-relational memory task. In the relational tasks (see Fig. $4 \mathrm{a}-\mathrm{c}$ ), successful relational memory required the formation and recollection of object-location or object-object relationships between the separate spatial or nonspatial components of an episode. In the non-relational memory task (Fig. 4d,e), successful performance did not require the memory of relations but could be accomplished on the basis of the familiarity of the spatial orientations of items.

\section{Relational memory}

\section{Study phase (Fig. 4a)}

For each participant, 72 objects were randomly paired to create the 36 study items. These 36 item pairs were shown in one of 18 distinct spatial configurations in an invisible $8 \times 6$ grid. Following the original study, each spatial configuration was used twice in two different object pairings, which allowed altering the object-object relationships without a simultaneous change in object-location relationships. The participants were asked to memorize the object-object and object-location relationships of each pair and were shown examples of old and new test items in the spatial and nonspatial relational conditions, so that they would be able to focus on the relevant features. Each item was displayed for $2000 \mathrm{~ms}$ each with ISIs of $3000 \mathrm{~ms}$. The 36 items were presented in blocks of four (30 sec between-block intervals); each pair appeared only once within a block. The recognition test followed immediately after the fourth repetition of the study phase.

\section{Test of spatial relational memory (Fig. 4b)}

Each of the 36 old and 18 novel items was randomly presented for $2500 \mathrm{~ms}$ with an ISI of $3000 \mathrm{~ms}$. Old and new items were made up of the same object pairs. In old items, the spatial arrangement of the two objects was identical to the study phase; in novel items, the spatial positions of the objects were swapped. Participants were asked to indicate by button-presses whether an item was old or novel. The discrimination index $\mathrm{P}_{\mathrm{r}}$ and bias index $\mathrm{B}_{\mathrm{r}}$ of the Two-High-Threshold Model $\left(\mathrm{P}_{\mathrm{r}}=\right.$ hit rate - false alarm rate, and $B_{r}=$ false alarm rate/[1 - (hit rate - false alarm rate)] were calculated to estimate spatial relational memory. The TwoHigh-Threshold Model with $\mathrm{P}_{\mathrm{r}}$ and $\mathrm{B}_{\mathrm{r}}$ as a dependent measure was chosen, because it was reported to be more sensitive than the signal-detection theory discrimination measure (Snodgrass and Corwin 1988). RTs were also recorded.

\section{Test of nonspatial relational memory (Fig. 4c)}

The 36 old and 18 novel items were presented in random order; items were presented for $2500 \mathrm{~ms}$ each, with ISIs of $3000 \mathrm{~ms}$. All items retained the same locations on the screen as in the study phase, but they differed with respect to nonspatial pairing: Old items entailed the same object pairs as in the study phase, whereas novel items involved new object combinations, with each object keeping its spatial location from the study phase. Participants had to indicate by button-presses whether an item was old or novel, and discrimination and bias indices as well as RTs were assessed.

\section{Non-relational memory}

\section{Study phase (Fig. 4d)}

Thirty-six item pairs were presented at the central location of the screen. Subjects were asked to memorize the object-object relationships and the views from which the objects were displayed and were shown examples of old and new test items, so that they would be able to attend to the relevant features. As in the relational memory tasks, items were presented for $2000 \mathrm{~ms}$ with ISIs of $3000 \mathrm{~ms}$.

\section{Test of non-relational memory (Fig. 4e)}

The 36 old and 18 new items were presented in random order; each item was shown for $2500 \mathrm{~ms}$ with ISIs of $3000 \mathrm{~ms}$. All object pairs were shown at the same location as in the study phase. Old items preserved the view from which the objects were depicted. In novel items the objects were shown from a different view. Subjects had to indicate by button-presses whether an item was old or novel, and discrimination and bias indices and RTs were analyzed.

The order of the relational and non-relational memory tasks was randomized across subjects. There was only one study phase for the two relational tasks (see above), and the order of the spatial and nonspatial recognition phase was also randomized. Stimuli and responses were displayed, recorded, and analyzed using the Presentation software (Neurobehavioral Systems, Inc., http://www.neurobs.com/), and statistical analyses were performed via SPSS 15.0.

\section{Acknowledgments}

We thank Sabine Bierstedt for figure preparation, and are very grateful for helpful comments by reviewers on an earlier manuscript. E.S. is a scholarship recipient of the International Graduate School of Neuroscience, Bochum, Germany, and fellow of the Research School, Bochum, Germany.

\section{References}

Aggleton, J.P. and Brown, M.W. 1999. Episodic memory, amnesia and the hippocampal-anterior thalamic axis. Behav. Brain Sci. 22: 425489.

Aggleton, J.P. and Brown, M.W. 2006. Interleaving brain systems for episodic and recognition memory. Trends Cogn. Sci. 10: 455-463.

Buckley, M.J. 2005. The role of the perirhinal cortex and hippocampus in learning, memory, and perception. Q. J. Exp. Psychol. B 58: 246268.

Burgess, N., Maguire, E.A., and O'Keefe, J. 2002. The human hippocampus and spatial and episodic memory. Neuron 35: 625-641.

Burke, S.N. and Barnes, C.A. 2006. Neural plasticity in the ageing brain. Nat. Rev. Neurosci. 7: 30-40.

Bussey, T.J. and Saksida, L.M. 2005. The perceptual-mnemonic/feature conjunction model of perirhinal cortex function. Q. J. Exp. Psychol. B 58: $269-282$.

Bussey, T.J., Saksida, L.M., and Murray, E.A. 2002. Perirhinal cortex resolves feature ambiguity in complex visual discriminations. Eur. J. Neurosci. 15: 365-374.

Cabeza, R., Daselaar, S.M., Dolcos, F., Prince, S.E., Budde, M., and Nyberg, L. 2004. Task-independent and task-specific age effects on brain activity during working memory, visual attention and episodic retrieval. Cereb. Cortex 14: 364-375.

Cohen, R.M., Szczepanik, J., McManus, M., Mirza, N., Putnam, K., Levy, J., and Sunderland, T. 2006. Hippocampal atrophy in the healthy is initially linear and independent of age. Neurobiol. Aging 27: 13851394.

Dahl, G. 1972. WIP-Reduzierter Wechsler Intelligenztest: Anwendung, Auswertung, Statistische Analysen, Normwerte. Hain, Meisenheim/ Glem, Germany.

Daselaar, S.M., Fleck, M.S., Dobbins, I.G., Madden, D.J., and Cabeza, R. 2006. Effects of healthy aging on hippocampal and rhinal memory functions: An event-related fMRI study. Cereb. Cortex 16: 1771-1782.

Eichenbaum, H. 2001. The hippocampus and declarative memory: Cognitive mechanisms and neural codes. Behav. Brain Res. 127: 199-207. 
Eichenbaum, H. 2004. Hippocampus: Cognitive processes and neural representations that underlie declarative memory. Neuron 44: $109-120$.

Eldridge, L.L., Engel, S.A., Zeineh, M.M., Bookheimer, S.Y., and Knowlton, B.J. 2005. A dissociation of encoding and retrieval processes in the human hippocampus. J. Neurosci. 25: 3280-3286.

Fenker, D.B., Schott, B.H., Richardson-Klavehn, A., Heinze, H.J., and Duzel, E. 2005. Recapitulating emotional context: Activity of amygdala, hippocampus and fusiform cortex during recollection and familiarity. Eur. J. Neurosci. 21: 1993-1999.

Gilbert, P.E., Kesner, R.P., and Lee, I. 2001. Dissociating hippocampal subregions: Double dissociation between dentate gyrus and CA1. Hippocampus 11: 626-636.

Grady, C.L., Maisog, J.M., Horwitz, B., Ungerleider, L.G., Mentis, M.J., Salerno, J.A., Pietrini, P., Wagner, E., and Haxby, J.V. 1994. Age-related changes in cortical blood flow activation during visual processing of faces and location. J. Neurosci. 14: $1450-1462$.

Hedden, T. and Gabrieli, J.D.E. 2004. Insights into the ageing mind: A view from cognitive neuroscience. Nat. Rev. Neurosci. 5: 87-112.

Huettel, S.A., Singerman, J.D., and McCarthy, G. 2001. The effects of aging upon the hemodynamic response measured by functional MRI. Neuroimage 13: 161-175.

Insausti, R., Insausti, A.M., Sobreviela, M.T., Salinas, A., and Martinez-Penuela, J.M. 1998a. Human medial temporal lobe in aging: Anatomical basis of memory preservation. Microsc. Res. Tech. 43: 8-15.

Insausti, R., Juottonen, K., Soininen, H., Insausti, A.M., Partanen, K., Vainio, P., Laakso, M.P., and Pitkanen, A. 1998b. MR volumetric analysis of the human entorhinal, perirhinal, and temporopolar cortices. AJNR Am. J. Neuroradiol. 19: 659-671.

Jager, T., Mecklinger, A., and Kipp, K.H. 2006. Intra- and inter-item associations doubly dissociate the electrophysiological correlates of familiarity and recollection. Neuron 52: 535-545.

Kesner, R.P. and Hopkins, R.O. 2006. Mnemonic functions of the hippocampus: A comparison between animals and humans. Biol. Psychol. 73: 3-18.

Kesner, R.P., Gilbert, P.E., and Barua, L.A. 2002. The role of the hippocampus in memory for the temporal order of a sequence of odors. Behav. Neurosci. 116: 286-290.

Kohler, S., Danckert, S., Gati, J.S., and Menon, R.S. 2005. Novelty responses to relational and non-relational information in the hippocampus and the parahippocampal region: A comparison based on event-related fMRI. Hippocampus 15: 763-774.

Kumaran, D. and Maguire, E.A. 2005. The human hippocampus: Cognitive maps or relational memory? J. Neurosci. 25: 7254-7259.

Kumaran, D., Hassabis, D., Spiers, H.J., Vann, S.D., Vargha-Khadem, F., and Maguire, E.A. 2007. Impaired spatial and non-spatial configura learning in patients with hippocampal pathology. Neuropsychologia 45: 2699-2711.

Lavenex, P.B., Amaral, D.G., and Lavenex, P. 2006. Hippocampal lesion prevents spatial relational learning in adult macaque monkeys. J. Neurosci. 26: $4546-4558$.

Madden, D.J., Turkington, T.G., Provenzale, J.M., Denny, L.L., Hawk, T.C., Gottlob, L.R., and Coleman, R.E. 1999. Adult age differences in the functional neuroanatomy of verbal recognition memory. Hum. Brain Mapp. 7: 115-135.

Madden, D.J., Whiting, W.L., Provenzale, J.M., and Huettel, S.A. 2004. Age-related changes in neural activity during visual target detection measured by fMRI. Cereb. Cortex 14: 143-155.

Maguire, E.A., Frackowiak, R.S., and Frith, C.D. 1997. Recalling routes around London: Activation of the right hippocampus in taxi drivers. J. Neurosci. 17: 7103-7110.

Maguire, E.A., Burgess, N., Donnett, J.G., Frackowiak, R.S., Frith, C.D., and O'Keefe, J. 1998. Knowing where and getting there: A human navigation network. Science 280: 921-924.

Manns, J.R., Hopkins, R.O., Reed, J.M., Kitchener, E.G., and Squire, L.R. 2003. Recognition memory and the human hippocampus. Neuron 37: 171-180.

Mayes, A.R., Holdstock, J.S., Isaac, C.L., Montaldi, D., Grigor, J., Gummer, A., Cariga, P., Downes, J.J., Tsivilis, D., Gaffan, D., et al 2004. Associative recognition in a patient with selective hippocampal lesions and relatively normal item recognition. Hippocampus 14: 763-784.

Mitchell, K.J., Johnson, M.K., Raye, C.L., and D'Esposito, M. 2000. fMRI evidence of age-related hippocampal dysfunction in feature binding in working memory. Brain Res. Cogn. Brain Res. 10: 197-206.

Montaldi, D., Spencer, T.J., Roberts, N., and Mayes, A.R. 2006. The neural system that mediates familiarity memory. Hippocampus 16: $504-520$.

Norman, K.A. and O'Reilly, R.C. 2003. Modeling hippocampal and neocortical contributions to recognition memory: A complementary-learning-systems approach. Psychol. Rev. 110: 611-646.

O'Keefe, J. 1999. Do hippocampal pyramidal cells signal non-spatial as well as spatial information? Hippocampus 9: 352-364.

Pihlajamaki, M., Tanila, H., Kononen, M., Hanninen, T., Hamalainen, A., Soininen, H., and Aronen, H.J. 2004. Visual presentation of novel objects and new spatial arrangements of objects differentially activates the medial temporal lobe subareas in humans. Eur. J. Neurosci. 19: 1939-1949.

Prull, M.W., Dawes, L.L.C., Martin, A.M., Rosenberg, H.F., and Light, L.L. 2006. Recollection and familiarity in recognition memory: Adult age differences and neuropsychological test correlates. Psychol. Aging 21: $107-118$.

Quamme, J.R., Yonelinas, A.P., and Norman, K.A. 2007. Effect of unitization on associative recognition in amnesia. Hippocampus 17: $192-200$.

Raz, N., Lindenberger, U., Rodrigue, K.M., Kennedy, K.M., Head, D., Williamson, A., Dahle, C., Gerstorf, D., and Acker, J.D. 2005 Regional brain changes in aging healthy adults: General trends, individual differences and modifiers. Cereb. Cortex 15: 1676-1689.

Rolls, E.T. and Kesner, R.P. 2006. A computational theory of hippocampal function, and empirical tests of the theory. Prog. Neurobiol. 79: 1-48.

Rossion, B. and Pourtois, G. 2004. Revisiting Snodgrass and Vanderwart's object pictorial set: The role of surface detail in basic-level object recognition. Perception 33: 217-236.

Rugg, M.D. and Yonelinas, A.P. 2003. Human recognition memory: A cognitive neuroscience perspective. Trends Cogn. Sci. 7: 313-319.

Rutishauser, U., Mamelak, A.N., and Schuman, E.M. 2006. Single-trial learning of novel stimuli by individual neurons of the human hippocampus-amygdala complex. Neuron 49: 805-813.

Schiltz, K., Szentkuti, A., Guderian, S., Kaufmann, J., Munte, T.F. Heinze, H.J., and Duzel, E. 2006. Relationship between hippocampal structure and memory function in elderly humans. J. Cogn. Neurosci. 18: $990-1003$

Snodgrass, J.G. and Corwin, J. 1988. Pragmatics of measuring recognition memory: Applications to dementia and amnesia. J. Exp. Psychol. Gen. 117: 34-50.

Spiers, H.J., Maguire, E.A., and Burgess, N. 2001. Hippocampal amnesia. Neurocase 7: 357-382.

Toth, J.P. and Parks, C.M. 2006. Effects of age on estimated familiarity in the process dissociation procedure: The role of noncriterial recollection. Mem. Cognit. 34: 527-537.

Wais, P.E., Wixted, J.T., Hopkins, R.O., and Squire, L.R. 2006. The hippocampus supports both the recollection and the familiarity components of recognition memory. Neuron 49: 459-466.

Walhovd, K.B., Fjell, A.M., Reinvang, I., Lundervold, A., Dale, A.M., Eilertsen, D.E., Quinn, B.T., Salat, D., Makris, N., and Fischl, B. 2005. Effects of age on volumes of cortex, white matter and subcortical structures. Neurobiol. Aging 26: 1261-1270.

Wechsler, D. 1987. Wechsler Memory Scale-Revised. The Psychological Corporation, London, UK.

Wixted, J.T. 2007. Dual-process theory and signal-detection theory of recognition memory. Psychol. Rev. 114: 152-176.

Wixted, J.T. and Squire, L.R. 2004. Recall and recognition are equally impaired in patients with selective hippocampal damage. Cogn. Affect. Behav. Neurosci. 4: 58-66.

Yonelinas, A.P. 2002. The nature of recollection and familiarity: A review of 30 years of research. J. Mem. Lang. 46: 441-517.

Yonelinas, A.P., Kroll, N.E., Quamme, J.R., Lazzara, M.M., Sauve, M.J., Widaman, K.F., and Knight, R.T. 2002. Effects of extensive temporal lobe damage or mild hypoxia on recollection and familiarity. Nat. Neurosci. 5: 1236-1241.

Received August 30, 2007; accepted in revised form November 1, 2007. 


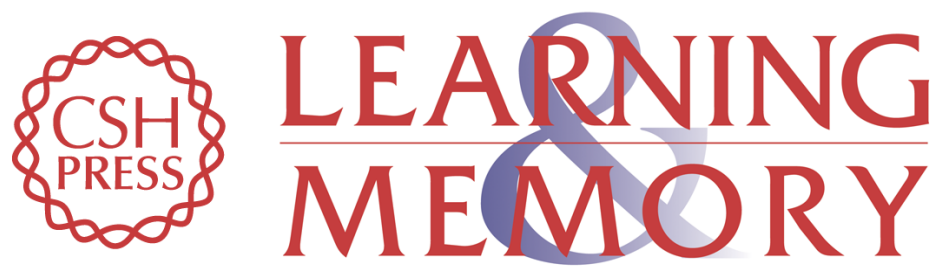

\section{Course of relational and non-relational recognition memory across the adult lifespan}

Eleonore Soei and Irene Daum

Learn. Mem. 2008, 15:

Access the most recent version at doi:10.1101//m.757508

References This article cites 55 articles, 6 of which can be accessed free at: http://learnmem.cshlp.org/content/15/1/21.full.html\#ref-list-1

License

Email Alerting Receive free email alerts when new articles cite this article - sign up in the box at the Service top right corner of the article or click here. 